\title{
A Paper-Based “Pop-up” Electrochemical Device for Analysis of Beta-Hydroxybutyrate
}

\section{Citation}

Wang, Chien-Chung, Jonathan W. Hennek, Alar Ainla, Ashok A. Kumar, Wen-Jie Lan, Judy Im, Barbara S. Smith, Mengxia Zhao, and George M. Whitesides. 2016. A Paper-Based 'Pop-up' Electrochemical Device for Analysis of Beta-Hydroxybutyrate. Analytical Chemistry 88, no. 12: 6326-6333. doi:10.1021/acs.analchem.6b00568.

\section{Published Version}

10.1021/acs.analchem.6b00568

\section{Permanent link}

http://nrs.harvard.edu/urn-3:HUL.InstRepos:29953908

\section{Terms of Use}

This article was downloaded from Harvard University's DASH repository, and is made available under the terms and conditions applicable to Open Access Policy Articles, as set forth at http:// nrs.harvard.edu/urn-3:HUL.InstRepos:dash.current.terms-of-use\#OAP

\section{Share Your Story}

The Harvard community has made this article openly available.

Please share how this access benefits you. Submit a story.

\section{Accessibility}




\section{A Paper-Based "Pop-up" Electrochemical Device for Analysis of Beta-Hydroxy- butyrate}

Chien-Chung Wang ${ }^{1}$, Jonathan W. Hennek ${ }^{1}$, Alar Ainla ${ }^{1}$, Ashok A. Kumar ${ }^{1}$, Wen-Jie Lan ${ }^{1}$, Judy Im $^{1}$, Barbara Smith $^{1}$, Mengxia Zhao ${ }^{1}$ and George M. Whitesides ${ }^{1,2,3^{*}}$

${ }^{1}$ Department of Chemistry \& Chemical Biology, Harvard University, 12 Oxford Street, Cambridge, MA 02138, USA

${ }^{2}$ Wyss Institute for Biologically Inspired Engineering, Harvard University, 60 Oxford Street, Cambridge, MA 02138, USA

${ }^{3}$ Kavli Institute for Bionano Science \& Technology, Harvard University, 29 Oxford Street, Cambridge, MA 02138, USA

*Corresponding author E-mail: gwhitesides@ gmwgroup.harvard.edu 


\section{ABSTRACT}

This paper describes the design and fabrication of a "pop-up" electrochemical paper-based analytical device (pop-up-EPAD) to measure beta-hydroxybutyrate (BHB) — a key biomarker for diabetic ketoacidosis - using a commercial glucometer. Pop-up-EPADs are inspired by pop-up greeting cards and children's books. They are made from a single sheet of paper folded into a threedimensional (3D) device that changes shape, and fluidic and electrical connectivity, by simply folding and unfolding the structure. The reconfigurable 3D structure makes it possible to change the fluidic path and to control timing; it also provides mechanical support for the folded and unfolded structures that enables good registration and repeatability on folding. A pop-up-EPAD designed to detect $\mathrm{BHB}$ shows performance comparable to commercially available plastic test strips over the clinically relevant range of BHB in blood when used with a commercial glucometer that integrates the ability to measure glucose and BHB (combination BHB/glucometer). With simple modifications of the electrode and fluid path design, the pop-up-EPAD also detects BHB using a simple glucometer - a device that is much more available than combination BHB/glucometers. Strategies that use a "3D pop-up" — that is, large-scale changes a 3D structure and fluidic pathsby folding/unfolding add functionality (e.g., controlled timing, fluidic handling and path programming, control over complex sequences of steps, and alterations in electrical connectivity) to EPADs, and should enable the development of new classes of paper-based diagnostic devices.

Keywords: Pop-up structure, Kirigami, point-of-care diagnostics, clinical chemistry, electroanalytical chemistry, paper diagnostics, beta-hydroxybutyrate (BHB), diabetic ketoacidosis (DKA), diabetes 


\section{INTRODUCTION}

Nearly 400 million people have diabetes, $80 \%$ of whom live in low- and middle-income countries (LMICs). ${ }^{1}$ Uncontrolled diabetes can lead to the catabolism of fatty acids, and the production of so-called "ketone bodies," comprising acetone (2\%), acetoacetate (AcAc, 20\%), and beta-hydroxybutyrate (BHB, $78 \%)^{2}$ The build-up of these metabolic acids can cause an acid-base imbalance called "diabetic ketoacidosis" (DKA). ${ }^{3}$ Without early detection and treatment, DKA can be fatal. Despite improvements in insulin therapy from the 1980's to early 2000 's, mortality rates for DKA in developed countries has remained stubbornly high ( $4 \%$ of people with DKA die from it). ${ }^{2,4,5}$ In the developing world, although limited medical records make exact numbers difficult to establish, the mortality rate from DKA in diabetics is thought to be even higher. ${ }^{4,6}$ The use of point-of-care (POC) diagnostic tools for the early detection of DKA presents an opportunity to identify and treat DKA before it reaches acute levels. ${ }^{2,3,7}$ Direct measurement of BHB in blood is the best method for diagnosing DKA. ${ }^{7,8}$

Electrochemical enzymatic detection of BHB has recently been enabled by hand-held devices that use disposable test strips and that can be used at the POC. ${ }^{9-12}$ These hand-held detectors have the advantages of quantitative detection and simple integration with datamanagement systems and "the cloud". They are, however, less readily available, and more expensive, than commercial glucometers. Most glucometers cannot be used to read current test strips for BHB, because the timing for the multi-step enzymatic reaction used to measure BHB is different than that used to measure glucose. The test strips used for BHB are also expensive (\$58 each), in part because they require both complex fabrication to enable the multi-step sequence of enzymatic reactions required to detect $\mathrm{BHB}$, and relatively large quantities of expensive biochemical reagents to give short assay times. ${ }^{10}$ The requirement that patients purchase a meter 
specifically to measure BHB, and the cost of the strips, prevents widespread use even in developed countries. ${ }^{13}$ A low-cost device that could be read by glucometers could reduce the barriers to BHB monitoring.

This paper describes the development of a prototype of an inexpensive, paper-based test strip for the detection of BHB that operates using a commercial glucometer. In order to accommodate the multi-step reaction to detect BHB, we have developed a new type of device: a reconfigurable three-dimensional (3D) "pop-up" structure inspired by pop-up greeting cards

(Figure 1). This structure provides the basis of an electrochemical paper-based analytical device (pop-up-EPAD). The reconfigurability of the flow path in this device that can be achieved by folding and unfolding, and the ability to separate and reconnect layers spatially in the pop-up structure, enables time-controlled valving of fluid flow in a simple device made from one sheet of folded paper.

We demonstrate the feasibility of this pop-up-EPAD by testing whole blood spiked with $\mathrm{BHB}$, and find good agreement between paper devices measured by a reader for glucose, and commercial test strips measured by a reader for $\mathrm{BHB}$, throughout the clinically relevant range from $0.1 \mathrm{mM}$ to $6.0 \mathrm{mM}$. By creating a low-cost device for BHB from a single sheet of paper, and by demonstrating that this device can be read by a common, commercial electronic technology (i.e., a glucometer), we hope to improve access to diagnostics for DKA at the POC, and thus improve management of diabetes.

\section{Paper Provides an Excellent Platform for Electrochemical Analysis}

Paper-based microfluidic devices can be used for performing chemical analyses using hand-held devices. ${ }^{14-20}$ Paper devices are less expensive, and easier to fabricate than openchannnel microfluidic chips (normally fabricated in polymers), and do not require pumps and 
Figure 1. An illustration of the process of testing BHB using "pop-up" paper test strips and a glucometer.
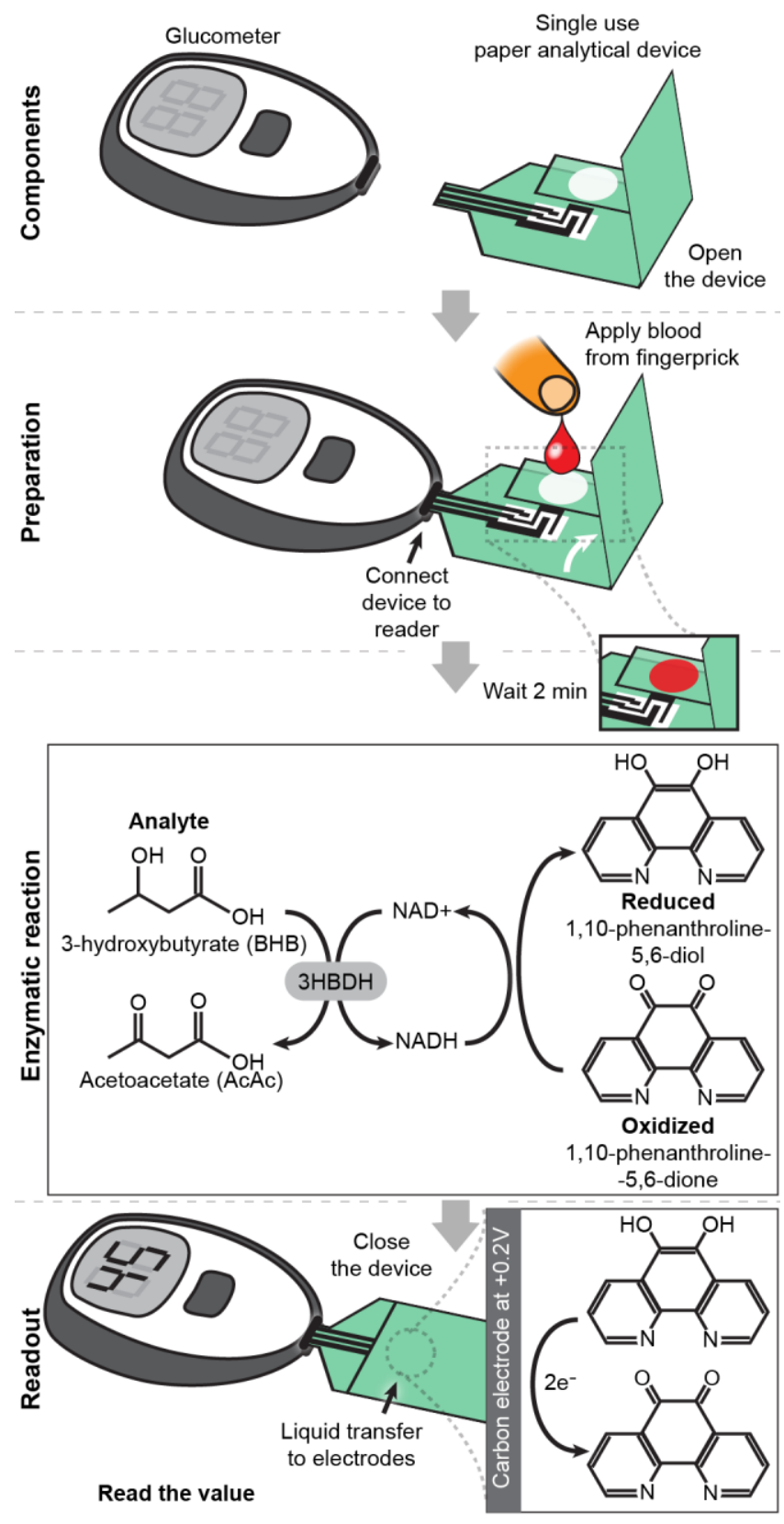
electrical power to manipulate fluids. The reagents necessary for chemical analyses (e.g., enzymes, substrates, and electrochemical mediators or colormetric indicators) can be stored in the pores between the cellulose fibers of the paper either as a dry powder (usually included in a solid stablizing agent such as dextran or trehalose), or suspended in a hydrogel; there is thus very little manipulation required by the users. To run a complete assay, the user simply adds the sample and a solution of electrochemical mediator (alternatively, the mediator can be stored in the device) to the popped up (unfolded) device, inserts the device into a glucometer, waits for a short time, folds it down, and reads the results. (Paper devices also have disadvantages relative to other type of microfluidic systems - complex paths for flow, limited control of flow rates and volumes, evaporation of water and changes in concentration, and optically scattering medium, and others. The relative merit of paper- and polymeric devices depends on the application. This paper is intended to address weaknesses in paper devices in timing and control of flow paths.)

Fluid flow can be controlled in a paper-based microfluidic device by wax barriers (i.e. channels) patterned using a solid-wax printer. We and others have used paper-based microfluidic devices for a wide range of biological and environmental applications. ${ }^{18-25}$ Paper-based devices are now of increasing interest for the electrochemical detection of analytes, ${ }^{26-29}$ in part for the ease with which their results can be transferred to the cloud, or to a distant clinic or doctor's office. Electrochemistry offers three advantages as the basis for bioanalysis: i) It provides quantitative measurements. ii) It is independent of lighting and color (both good lighting and a colorless solution are usually required for colorimetric and spectrophotometric assays). iii) It allows easy interfacing with electronic medical-records systems.

We and others have developed electrochemical paper-based analytical devices (EPADs) by stencil-printing carbon or silver onto wax-printed paper devices to make working, counter, 
and reference electrodes. ${ }^{26,28,30-35}$ We have also incorporated ion-selective membranes into these devices to make ion-selective electrodes. ${ }^{36}$ These EPADs can perform a wide range electrochemical methods (e.g., potentiometry, voltammetry, chronoamperometry, and coulometry) to detect a variety of analytes. . $^{14,32,36-44}$

In order to apply EPADs in POC settings, we have integrated the devices with a commercially available glucometer, ${ }^{14}$ and also developed a much more versatile, portable, electrochemical reader capable of a wide variety of electrochemical measurements with transmission of data over the audio channel of any cell-phone, with any mobile network..$^{37,45}$

One of the limitations of previous EPADs is that the enzymes and other reagents are stored on or near the electrodes. When the solution of sample or mediator contacts the electrodes and the EPAD is connected to a commercial glucometer (or other commercial device), the electronics immediately start the measurement (sometimes after only a $5-10$ s reaction period). This automatic (and immediate) electrical response limits the time allowed for a reaction. In order to generate a measureable signal within these time limits, we, and others, have increased the concentration of the enzyme stored in the device, but using more enzyme also increases cost. ${ }^{14,17,27}$ For some applications, there are no enzymes that can react rapidly enough to work with conventional EPADs. To overcome these challenges, a device must be able to decouple the enzymatic reaction from the specific timing sequence for analysis imposed by commercial glucometers. 


\section{EXPERIMENTAL DESIGN}

\section{Design and Fabrication of the pop-up-EPAD}

We were inspired by the ability of pop-up greeting cards and children's books to change the topography and topology of 3D structures quickly and easily. With this inspiration, we have developed a new class of EPADs with a pop-up, 3D structure. The pop-up-EPAD (Figure 2 and Figure S1) includes a sample port, a reaction zone where enzymes can be stored, and a detection zone that is spatially separated from the first two zones. The detection zone interfaces with a glucometer, through three stencil-printed electrodes: i) a working electrode, ii) a common counter and reference electrode, and iii) an indicator electrode. The pop-up structure acts as a reversible, mechanical valve to change the fluidic connectivity of the system. When the device is 'closed' using a modest mechanical pressure (i.e., when squeezed between the thumb and forefinger or placed on a flat surface with a weight on top), the valve goes from an 'off' to an 'on' state because the contact between the separate paper components allows a fluid connection, with liquid flowing from one sheet to another (Figure 2c). This connection is insensitive to the applied pressure, since it requires, primarily, fluidic contact and capillarity to establish the fluidic path, not consistent reproducible mechanical contact between the surfaces of the paper (Supporting Information, Figure S2).

The fabrication of a pop-up-EPAD requires six steps: i) wax-printing microfluidic channels on chromatographic paper (Whatman $1 \mathrm{Chr}),{ }^{46}$ ii) stencil-printing electrodes and circuits using graphite ink, iii) pipetting a solution of enzyme and cofactor into the reaction zone, iv) drying the system for six hours at $4^{\circ} \mathrm{C}$ in the dark, v) cutting the paper with a razor along printed lines, and vi) folding the pop-up paper device (Figure S3 and Figure S4). The first five steps of the fabrication process can be performed on a single sheet of paper, and could be carried out on a reel- 
Figure 2 (a) Photographs of a pop-up greeting card, and a pop-up structure of the type used in the EPAD described here. (b) A schematic of the pop-up-EPAD in the 'open' format. Enzymes are stored in the reaction zone. The detection zone contains printed electrodes designed specifically for the commercial electrochemical reader used in this study. IE: indicator (filling) electrode; CE/RE: common counter and reference electrode; WE: working electrode. (c) Photographs showing the valve capabilities of the pop-up-EPAD when it is open and closed. 

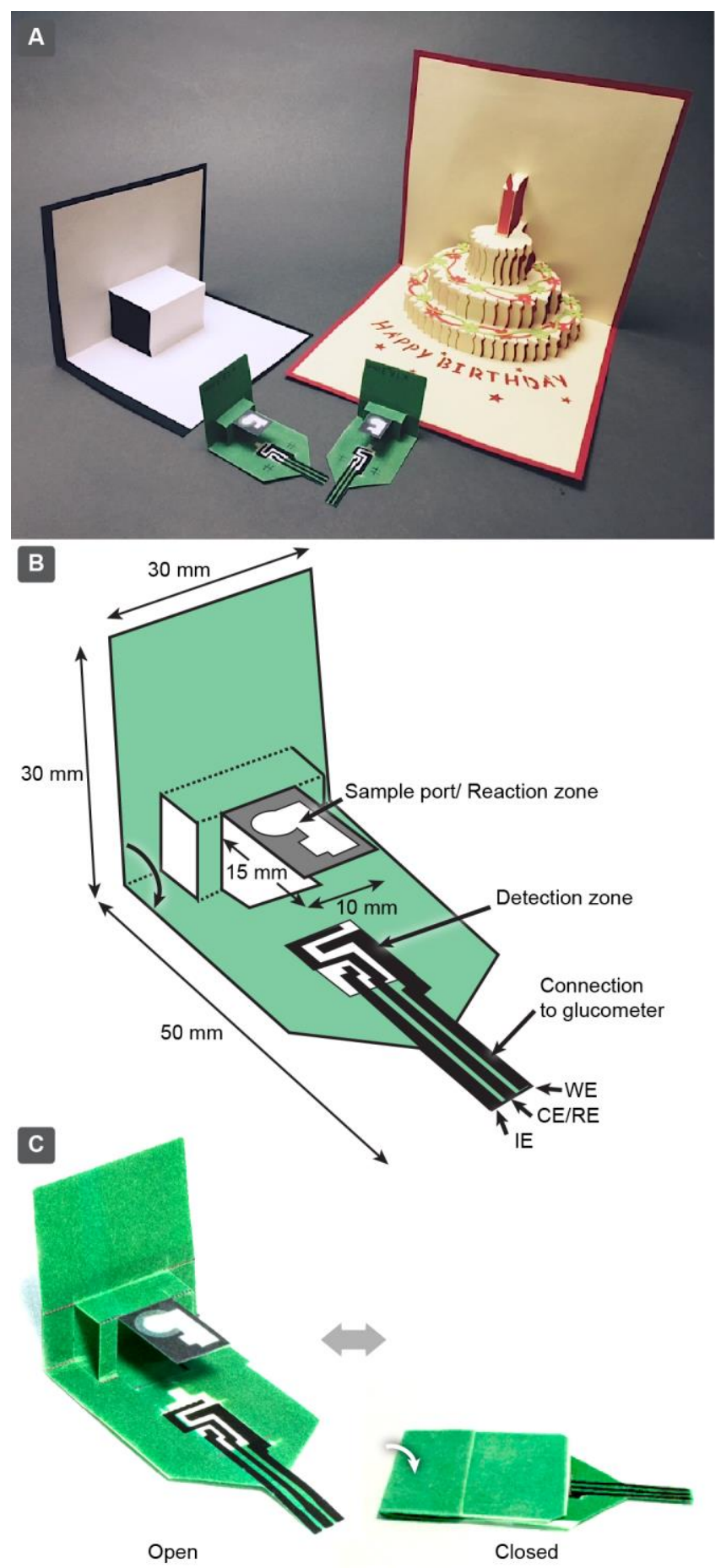
to-reel printing line or digital craft-cutting plotters. ${ }^{47}$ After the individual test strips are separated, a series of "through cuts" (i.e., a cut through the entire thickness of the paper) and "half cuts" (i.e., a light cut that does not go through the entire thickness of the paper but enables easy folding) are performed over guiding lines. The simple and reproducible fabrication procedure ensures that the devices that are produced have consistent overlap between the reaction zone and detection zone. The Supporting Information includes a step-by-step video of the entire fabrication process, and a PDF giving the design — including a guide to cutting and folding the pop-up structure — used to print the devices.

\section{Choice of Glucometer: Precision Xtra ${ }^{\circledR}$ Meter}

We used the Precision Xtra ${ }^{\circledR}$ Blood Glucose Monitoring System (Abbott Laboratories Inc.) as the electrochemical reader that combines the ability to measure glucose and BHB. This glucometer has three attractive characteristics: i) it is relatively low in cost ( $\$ 35$ for the device) and easy to use; ii) it is one of only two commercial glucometers that also measures the concentration of BHB; and iii) it has been widely used in hospital and field tests, adequately characterized in the literature, and demonstrated to have reliable performance in human and animal studies. ${ }^{48}$ The Precision Xtra ${ }^{\circledR}$ meter uses different test strips for glucose and for BHB; the device recognizes the type of strip automatically based on a recognition electrode on the back side of the BHB strip. That is, the glucose strips have no electrodes on the backside; the BHB strips have a recognition electrode patterned onto their backside (see images of deconstructed electrodes in Figure S5).

We aimed to create a device for BHB that could be read by simple glucose meters without requiring this type of recognition of strips; we, therefore, designed our paper devices without 
the recognition electrode on the backside. The pop-up-EPADs for detecting BHB mimic the configuration of commercial glucose strips in term of the configuration at the junction between the strip and the meter, and, thus, trick the meter into operating as a glucometer. We also designed strips that could be used with a commercial glucometer (CVS Truetrack ${ }^{\circledR}$ glucometer), which do not include a mode for measuring BHB (Figure S6 and Figure S7). In both cases, the number displayed by the reader is a representation of the actual BHB concentration value and not the exact concentration; the true value of $\mathrm{BHB}$ can be calculated from the reading on the meter using a calibration curve, or the reading for a pop-up-EPAD can be read against an empirical scale. Neither is satisfactory for a fully developed device, but for a prototype, the design we used is sufficient. In a device intended for POC use, the conversion could be easily accomplished by the electrochemical reader.

Although we only demonstrate readings with two different commercial meters in this work, the pop-up-EPADs could be used with other glucometers and electrochemical detectors with simple modifications of the electrodes, and other design aspects required to generate the electrochemical interface designed for that device. Using the Precision $\mathrm{Xtra}^{\circledR}$, we were able to make a direct comparison of our measurements of BHB, made with paper devices, to those made with commercial, plastic test strips using the same reader in different test modes.

\section{The Pop-up-EPAD Enables Controlled Valving and Timing}

The pop-up structure provides spatial separation in the "open" configuration; it enables the operator to wait for the enzymatic reaction to reach completion before changing the path of the fluid (i.e. 'closing' the pop-up) and enabling the fluid flow that triggers the initiation of the electrochemical measurement sequence by the glucometer. 
One challenge in lowering the cost of POC detection of BHB is the high cost of the enzyme, 3-hydrozybutyrate dehydrogenase (3-HBHD, EC 1.1.1.30; \$5-7/U); the smaller the amount of enzyme used in the reaction, the longer the time required for the assay to reach completion. Previous work in our lab and elsewhere using EPADs to detect blood glucose used $\geq 0.5$ $\mathrm{U}$ of glucose oxidase/device compared to $\geq 0.03 \mathrm{U} /$ device for commercial strips. ${ }^{14,17,27}$ Glucose oxidase is, however, much less expensive than 3-HBHD ( $\$ 0.0058 / \mathrm{U}$ vs. $\sim \$ 5-7 / \mathrm{U})$. The pop-upEPAD structure enables the controlled timing of the enzymatic reaction. We can, therefore, reduce the total amount of the 3-HBHD required by allowing a longer reaction time, and thus reduce the cost of each test strip (with the trade-off being that the analysis is slower). This design also allows reagents to be stored in the paper and to be activated by the fluid flow; no premixing of the components is needed.

\section{Enzymatic Reaction Principle}

We used a commercially available BHB assay kit (Randox Laboratories, Inc) that contains 3-HBDH and $\mathrm{NAD}^{+}$. Using this kit, we designed the amperometric assay for $\mathrm{BHB}$ on the pop-up-EPAD to have three steps (Figure 1 and Figure S8): i) 3-HBDH catalyzes the oxidation of BHB (present in the sample) to acetoacetate (AcAc), with a corresponding reduction of $\mathrm{NAD}^{+}$to NADH; ii) the NADH produced donates two electrons to the electron-transfer mediator, 1,10-phenanthroline-5,6-dione (1,10-PD), and generates the reduced form of 1,10-PD; ${ }^{9}$ iii) the working electrode oxidizes the reduced form of 1,10-PD at a potential of $+0.2 \mathrm{~V}$ (set automatically by the hand-held reader), and the resulting current is displayed as a numerical quantity on the electrochemical reader. We varied the quantity of enzyme and the cofactor $\mathrm{NAD}^{+}$ to ensure that the signal for appropriate BHB concentrations would correspond to the linear 
range of the glucose output; if a concentration were out of this range, the reader would display an out-of-range error message rather than a number.

\section{RESULTS AND DISCUSSION}

\section{Ensuring Performance of the Carbon Electrodes}

In order to test the performance of stencil-printed carbon electrodes, we first performed cyclic voltammetry on a solution of the mediator, 1,10-PD. We scanned the voltage between -0.3 and $0.6 \mathrm{~V}$, at a scan rate of $50 \mathrm{mV} / \mathrm{s}$, in the presence of different concentrations of NADH. Figure S9 shows a concentration-dependent increase in the height of the anodic peak in a mixed solution of 1,10-PD and NADH. The dependence of peak current on the concentration of NADH demonstrates that stencil-printed carbon electrodes on paper behave similarly to the screenprinted electrodes on plastic test strips.

\section{Integration of the pop-up-EPAD with glucometer}

We developed an EPAD with a "pop-up" format that allows an enzymatic assay for BHB to be read with a commercial glucometer. First, we prepared the pop-up-EPADs having the enzyme and cofactor reagents stored on the devices. An enzyme/cofactor solution was prepared to a final concentration of $2 \mathrm{U} / \mathrm{mL}$ of $3-\mathrm{HBDH}$ and $42 \mathrm{mM} \mathrm{NAD}^{+}$in Tris-buffer $(\mathrm{pH} 8.0)$, spotted onto the reaction zone of pop-up-EPADs, and dried at $4{ }^{\circ} \mathrm{C}$ for six hours in the dark (Supporting Information). The volume of the enzyme/cofactor solution was chosen to be $45 \mu \mathrm{L}$ by titration to ensure that the signal for appropriate BHB concentrations would correspond to the linear range of the glucose output (Table S1). Figure S10a-c shows the operation of the device with the glucometer. First, we inserted the dry pop-up-EPAD into the glucometer in the open configuration and waited for the reader to indicate it recognized the device. Then we loaded the 
sample (BHB in buffer) and a separate mediator solution $(2.5 \mathrm{mg} / \mathrm{mL} 1,10-\mathrm{PD})$ onto the reaction zone in the top layer of paper device. The sample fluid was retained in the top layer of paper, and the bottom layer remained dry (because there is no fluidic connection between the two zonestop and bottom - in the "open" configuration). After the enzymatic reaction was completed (at a speccified time based on the level of enzymatic activity in the devices), we changed the fluidic

connectivity by simply closing the device. The liquid from the reaction zone could then wick into the detection zone. Once the sample reached the electrodes, the glucometer initiated the amperometric measurement at a potential of $+0.2 \mathrm{~V}$ and displayed a number for the measured analyte.

We demonstrated the use of the Precision Xtra ${ }^{\circledR}$ reader in glucometer mode to analyze the concentrations of BHB in Tris buffer (100 mM Tris-HCI, $\mathrm{pH}$ 8.0). The normal range of BHB in healthy individuals is less than 0.4 to $0.5 \mathrm{mM}$, and diabetics with a BHB concentration greater than $3 \mathrm{mM}$ are advised to seek medical attention immediately. ${ }^{2,3}$ The curve for the measurement of BHB shows that the values displayed were linearly proportional to the BHB concentrations in the range of $0.1-6.0 \mathrm{mM}\left(\mathrm{R}^{2}=0.98\right.$, Figure $\left.\mathrm{S} 10 \mathrm{~d}\right)$. These data demonstrate that the electrode structure of the pop-up-EPAD can distinguish different concentrations of BHB in Tris buffer.

\section{Off-chip validation of the electrodes of the pop-EPAD with BHB measurement using whole}

\section{blood sample}

Conponents present in whole blood can have confounding effects on electrodes and on the accuracy of electrochemical assays. ${ }^{27,49}$ To evaluate this potential interference, we thus tested BHB-spiked whole blood mixed with reagents off-chip and found a linear response (Supporting Information, Figure S11). 


\section{Validation of the pop-EPAD with BHB measurement using whole blood sample}

We next tested the complete pop-up-EPAD system with dried enzyme and cofactor reagents stored in the device. We spotted $45 \mu \mathrm{L}$ of the enzyme/cofactor solution containing 2 $\mathrm{U} / \mathrm{mL}$ of $3-\mathrm{HBDH}$ and $42 \mathrm{mM} \mathrm{NAD}^{+}$in Tris-buffer onto the reaction zone. The devices were ready for use after the solution dried for six hours at $4{ }^{\circ} \mathrm{C}$ in the dark. To perform an assay, we added $15 \mu \mathrm{L}$ of BHB-spiked blood and $35 \mu \mathrm{L}$ of a separate mediator solution $(2.5 \mathrm{mg} / \mathrm{mL} 1,10$ PD) onto the reaction zone of the chip, where the reaction was allowed to proceed for two minutes, after which the device was closed. In order to ensure complete and reproducible wetting of the electrodes by the viscous whole blood, the device was held closed for 15-20 seconds with modest pressure $\left(>0.07 \mathrm{~N} / \mathrm{cm}^{2}\right)$. Figure 3 shows a linear response for BHB concentrations on both the pop-up-EPADs and the commercial test strips. The pop-up-EPADs display a good linear fit in the clinically relevant range of 0.1 to $6.0 \mathrm{mM}\left(\mathrm{R}^{2}=0.96\right)$. The limit of detection (LOD) was calculated to be the concentration that produced a display value three times the standard deviation displayed for a blank sample. While the commercial test strips result in a smaller standard deviation than our paper devices, the LOD values of our devices for BHB $(0.3 \mathrm{mM})$ were comparable to these of commercial test strips (0.12 $\mathrm{mM}$, Table 1$)$. Unlike the commercial test strips that were made in a manufacturing environment, the pop-up-EPADs were fabricated by hand in a laboratory. With additional automation and quality systems for manufacturing, the standard deviation for measurements with different test strips should decrease. Note that the value displayed by the glucometer was higher for measuring whole blood than it was for BHB in buffer. The increase in conductivity that caused this has been shown by many researchers, ${ }^{50,51}$ and is likely due to extraneous redox reactions between the mediator, 1,10-PD, and electro-active 
Figure 3. (a) Measurements of BHB spiked into whole blood with a pop-up-EPAD and a glucose meter. The dashed line represents a linear fit to the experimental data: $y=30 x+185.6\left(R^{2}=\right.$ 0.96) and error bars depict the standard deviation of replicate measurements $(n=7)(b)$ Measurements of BHB in human whole blood samples using three lots of commercially available test strips (Abbott, Precision Xtra ${ }^{\circledR}$ Blood Ketone Test Strip, LOT No. 75001, n =7, y = $1.01 \mathrm{x}+$ $0.03\left(\mathrm{R}^{2}=1.00\right)$ 
A
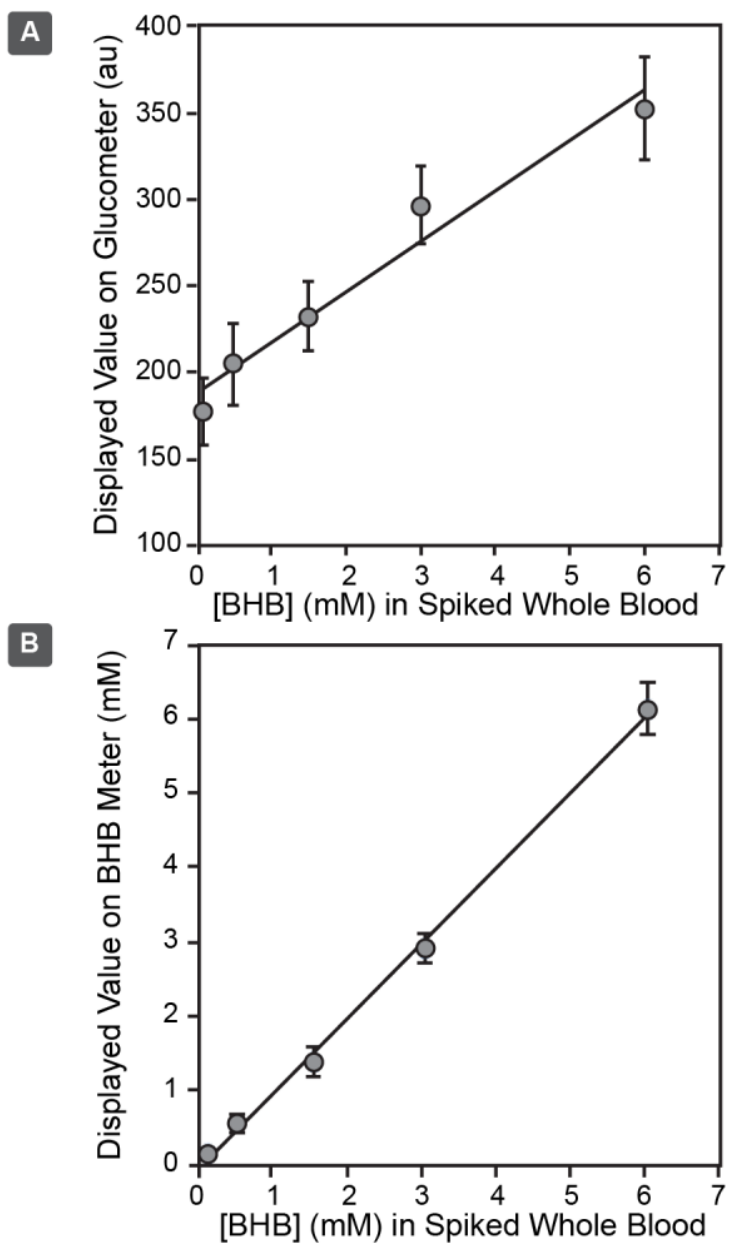
Table 1. Comparison of the performance of pop-up-EPADs with commercial, plastic BHB test strips

\begin{tabular}{lll} 
Substrate & $\begin{array}{l}\text { Commercial } \\
\text { Test }\end{array}$ & $\begin{array}{l}\text { Pop-up- } \\
\text { EPADs }\end{array}$ \\
\hline $\begin{array}{l}\text { Linear dynamic range } \\
(\mathrm{mM})\end{array}$ & 0 to 7 & 0 to 6 \\
$\begin{array}{l}\text { Limit of detection }(\mathrm{mM}) \\
\text { Minimum volume of }\end{array}$ & 0.12 & 0.3 \\
sample $(\mu \mathrm{L})$ & $\sim 2$ & $15-30$ \\
$\begin{array}{l}\text { Waiting time (seconds) } \\
\text { a }\end{array}$ & 10 & 120 \\
Price/Cost (dollars) & $\$ 5-8^{\mathrm{b}}$ & $\$ 0.521^{\mathrm{c}}$ \\
\hline
\end{tabular}

${ }^{a}$ The time interval between application of a sample to the test strip and reading the meter. ${ }^{\mathrm{b}}$ The price was determined based on the range of prices found on Amazon.com in the year 2015. ${ }^{\mathrm{c}}$ The cost is based on the cost of materials (See Table S2). The price of the commercial test strip cannot be compared with the cost of the materials of the pop-up device, since the corresponding cost for the commercial device will be less than its price. 
components of the whole blood (e.g., endogenous NADH-dehydrogenase, hemoglobin, glutathione, other proteins, ascorbic acid, uric acid and a mixture of charged amino acids, etc.). ${ }^{9}$

To elimilate the step of adding a separate mediator solution (a key consideration for practical use in the field), we developed an alternative approach to store the 1,10-PD mediator by spotting a detection zone in our devices, based on previous literature. ${ }^{9,14,52}$ We spotted $70 \mu \mathrm{L}$ of an aqueous solution of $0.5 \mathrm{mg} / \mathrm{mL} 1,10-\mathrm{PD}$ onto the detection zone, and allowed the device to dry for overnight at room temperature in the dark (1,10-PD in solution is sensitive to light). Next, $45 \mu \mathrm{L}$ of the enzyme and cofactor solution containing $2 \mathrm{U} / \mathrm{mL}$ of $3-\mathrm{HBDH}$ and $42 \mathrm{mM} \mathrm{NAD}^{+}$in Tris-buffer were added to the reaction zone. The devices were ready for use after the solution dried for six hours at $4{ }^{\circ} \mathrm{C}$ in the dark. With all of the reagents stored on the chip, the user simply adds $30 \mu \mathrm{L}$ of sample (BHB-spiked buffer or whole blood) onto the reaction zone, waits for two minutes, and closes the device to trigger the electrochemical analysis. The results based on this laboratory prototype were comparable with those obtained with commercial plastic test strips (Figure S12). By further optimizing the dimentions of the reaction/detection zones, electrode configuration, mediator/enzyme/cofactor concentrations, reaction timing and electrode sensitivity to hematocrit levels, ${ }^{27,53}$ it should be possible to reduce the volume of sample and improve performance. Recent advances in computer guided modeling and simulation using $\mathrm{COMSOL}^{\circledR}$ may enable accellerated optimization of these devices in the future. ${ }^{54}$

\section{CONCLUSION}

This paper describes a prototype of a new type of paper-based device- a "pop-upEPAD"- and demonstrates its application in measuring BHB at the POC using a commercially 
available glucometer. The integration of a pop-up structure into a paper device opens new opportunities in the design of paper-based diagnostic chips. The reconfigurable 3D structure makes it posible to change the fluidic path during the course of an analysis, and thus to control the timing of various steps. By controlling timing, we are able to reduce the concentration of enzymes, and the cost, compared to values reported previously. ${ }^{14,17,27}$ For certain applications, the pop-up structure might improve the dynamic range of measurements (relative to $2 \mathrm{D}$ test strips) by allowing controlled timing for reaction development and delivery of reagents in a multi-step programmed process. ${ }^{25,55}$

Pop-up-EPADs are fabricated in a conceptually simple process from a single sheet of paper (thus reducing the complexity and cost of farbrication). This work provides a new method to realize folding structures in paper-analytical devices of the type that we and others have developed for other applications. ${ }^{56-59}$ Origami, the transformation of a 2D piece of paper into a 3D structure (by folding without the use of cutting), and kirigami (which incorporates cutting) can provide methods to increase the capabilities of paper-based diagnostic chips, and to simplify fabrication and reduce their cost. ${ }^{26,31,38,40-42,60,61}$ Crooks et al. recently reported an improved fabrication technique utilizing origami folding to eliminate the requirement of using tape to secure layers in a paper-based diagnostic chip and the design of origami paper analytical devices (oPADs) for other applications. ${ }^{26,60,62-64}$ Our group has developed a folding analytical device based on treated hydrophobic and embossed paper that can be used to perform sequential incubation and washing steps. ${ }^{38}$ In these examples, folding is performed on a planar sheet of paper without the aid of mechanical supports to ensure good alignment between folded layers. The 3D structure of a pop-up-EPAD provides additional guidance for folding that should improve its registration and repeatability. 
Urine dipstick tests for AcAc are currently the cheapest test for "ketone bodies" in blood. These tests, however, do not measure BHB, the dominant ketone body in DKA. ${ }^{2,3}$ By the time AcAc concentration is elevated in the urine, ketoacidosis may already be severe, and the American Diabetes Association does not recommend the use of these dipstick-based tests. ${ }^{65,66}$ The current recommendation for POC monitoring of DKA is to measure BHB in the blood. Plastic test strips, and hand-held meters specifically designed to measure BHB, have been on the market for more than a decade. These tests provide an important tool to monitor DKA, but they remain so expensive (\$5-8 per test) that they are only used in high-risk patients, and they are non-routine even in the developed world. ${ }^{67,68}$

Most diabetic patients monitored for DKA already use a glucose meter. To monitor BHB in addition to glucose, these patients must either purchase an additional meter specialized for $\mathrm{BHB}$, or a specialized meter designed with modes that enable it to perform both glucose and BHB measurements. By developing what is effectively a valve using a pop-up structure, we have demonstrated a strategy that decouples the enzymatic reaction from the timing of the electrical sequence used by a commercial glucometer to carry out an electrochemical measurement of glucose. The pop-up-EPAD, when used for BHB and read with a simple glucometer, provides performance comparable (measured by linearity and sensitivity) to plastic test strips measured by a dedicated BHB-meter over the clinically relevant range of measurements $(0.1-6.0 \mathrm{mM})$. Because we use smaller quantities of enzymes than those used in the current design, ${ }^{14,17,27}$ the pop-up-EPAD takes more time (120 s) than commercial strips (10$30 \mathrm{~s})$ to perform a measurement. By using more enzyme, it would be possible to shorten the time, but the cost of reagents would increase. The cost of materials used to make a pop-up-EPAD is low (about $\$ 0.5$ per device; see Table S2), and the fabrication process can be carried out on a 
single sheet of paper. With production at a larger scale, these strips could provide an affordable test for BHB.

Measuring glucose in blood is the most common diagnostic measurement in the world; the average diabetic uses 764 test strips per year. ${ }^{69}$ While the number of patients with diabetic ketoacidosis (DKA) continues to increase,$^{70}$ the availability of BHB meters to diagnose DKA is still small, especially in LMICs, in part, because of the high cost of the commercial test strips. By integrating a pop-up structure into low-cost paper-based microfluidics, we have demonstrated a device that can, in principle, substitute more complex operation (in the unoptimized and incompletely developed prototype we describe) for (potentially) lower cost and greater accessibility, and that can be used, with minimal modifications, with ubiquitous glucometers.

For high-risk patients in LMICs, and moderate-risk patients everywhere, glucose meters are common, but BHB-meters are not as widely used. As a low-cost test that can plug into an existing glucometer, a pop-up-EPAD for BHB might provide a means to monitor DKA using meters that are already accessible to many patients with diabetes.

Integrating a pop-up structure into paper-based diagnostic devices provides more freedom and flexibility in design and use than previous devices made using the principle of origami. The 3D structure allows the path of the liquid flow, and of the electrical conductivity, to be reconfigured by spacially separating the layers by folding and unfolding the device. The concept of using a 3D pop-up structure provides five key functions for EPADs: i) controlling timing and enabling multistep processes; ii) providing good registration and repeatability upon folding; iii) interfacing with commercially avaliable hand-held glucometers for greater accessibility; iv) reducing the total amount of enzymes required (and, thus, reducing the cost) with the trade-off of 
increasing the time needed to make a measurement; and v) triggering the electrochemical mearsurment by folding.

In the furture, more elaborate versions of pop-up-structures can be made that include, for example, arbitrary fludic paths (e.g., multi-step fluidic programming during the course of an analysis) or other sensing components that respond to changes in electrical connectivity, optical and mechanical properties, magnetic fields, or chemical signals when folded/unfolded — and thus enable the development of new classes of paper-based devices.

\section{Acknowledgements}

This work was supported by an award from the Defense Threat Reduction Agency, HDTRA1-14-C-0037. C.W acknowledges the support by a postdoctoral fellowship from the Ministry of Science and Technology of Taiwan (R.O.C.). A.A. was supported by a postdoctoral fellowship from Swedish Research Council (VR), and A.A.K. was also supported by the U.S. National Science Foundation Graduate Research Fellowship Program.

\section{References}

(1) International Diabetes Federation. IDF Diabetes Atlas. 5 th ed. Brussels: International Diabetes Federation, 2013.

(2) Wallace, T. M.; Matthews, D. R. QJM-Int. J. Med. 2004, 97, 773-780.

(3) Laffel, L. Diabetes Metab. Res. Rev. 1999, 15, 412-426.

(4) Basu, A.; Close, C. F.; Jenkins, D.; Krentz, A. J.; Nattrass, M.; Wright, A. D. Diabetic Med. 1993, 10, 282-284. 
(5) Henriksen, O. M.; Røder, M. E.; Prahl, J. B.; Svendsen, O. L. Diabetes Res. Clin. Pract. 2007, 76, 51-56.

(6) Varadarajan, P. World J. Diabetes 2014, 5, 932-938.

(7) Arora, S.; Probst, M. A.; Agy, C.; Menchine, M. Diabetes Res. Clin. Pract. 2011, 94, 8688 .

(8) Sacks, D. B.; Arnold, M.; Bakris, G. L.; Bruns, D. E.; Horvath, A. R.; Kirkman, M. S.; Lernmark, A.; Metzger, B. E.; Nathan, D. M. Diabetes Care, 2011, 34, e61-e99.

(9) Forrow, N. J.; Sanghera, G. S.; Walters, S. J.; Watkin, J. L. Biosens. Bioelectron. 2005, 20, $1617-1625$.

(10) Fang, L.; Wang, S. H.; Liu, C. C. Sensor. Actuat. B Chem. 2008, 129, 818-825.

(11) Li, G.; Ma, N. Z.; Wang, Y. Sensor. Actuat. B Chem. 2005, 109, 285-290.

(12) Kwan, R. C. H.; Hon, P. Y. T.; Mak, W. C.; Law, L. Y.; Hu, J.; Renneberg, R. Biosens. Bioelectron. 2006, 21, 1101-1106.

(13) Guerci, B.; Tubiana-Rufi, N.; Bauduceau, B.; Bresson, R.; Cuperlier, A.; Delcroix, C.; Durain, D.; Fermon, C.; Le Floch, J.; Le Devehat, C.; Melki, V.; Monnier, L.; MosnierPudar, H.; Taboulet, P.; Hanaire-Broutin, H. Diabetes Metab. 2005, 31, 401-406.

(14) Nie, Z.; Deiss, F.; Liu, X.; Akbulut, O.; Whitesides, G. M. Lab Chip 2010, 10, 3163-3169.

(15) Delaney, J. L.; Hogan, C. F.; Tian, J.; Shen, W. Anal. Chem. 2011, 83, 1300-1306.

(16) Ellerbee, A. K.; Phillips, S. T.; Siegel, A. C.; Mirica, K. A.; Martinez, A. W.; Striehl, P.; Jain, N.; Prentiss, M.; Whitesides, G. M. Anal. Chem. 2009, 81, 8447-8452.

(17) Zhao, C.; Thuo, M. M.; Liu, X. Sci. Technol. Adv. Mater. 2013, 14, 054402

(18) Chin, C. D.; Linder, V.; Sia, S. K. Lab Chip 2012, 12, 2118-2134.

(19) Yetisen, A. K.; Akram, M. S.; Lowe, C. R. Lab Chip 2013, 13, 2210-2251.

(20) Cate, D. M.; Adkins, J. A.; Mettakoonpitak, J.; Henry, C. S. Anal. Chem. 2015, 87, 19-41. 
(21) Mao, X.; Huang, T. J. Lab Chip 2012, 12, 1412-1416.

(22) Pollock, N. R.; Rolland, J. P.; Kumar, S.; Beattie, P. D.; Jain, S.; Noubary, F.; Wong, V. L.; Pohlmann, R. A.; Ryan, U. S.; Whitesides, G. M. Sci. Transl. Med. 2012, 4, 152 ra129.

(23) Martinez, A. W.; Phillips, S. T.; Butte, M. J.; Whitesides, G. M. Angew. Chem. Int. Ed 2007, 46, 1318-1320.

(24) Yamada, K.; Henares, T. G.; Suzuki, K.; Citterio, D. Angew. Chem. Int. Ed. 2015, 54, 52945310.

(25) Lutz, B. R.; Trinh, P.; Ball, C.; Fu, E.; Yager, P. Lab Chip 2011, 11, 4274-4278.

(26) Liu, H.; Xiang, Y.; Lu, Y.; Crooks, R. M. Angew. Chem. Int. Ed. 2012, 51, 6925-6928.

(27) Noiphung, J.; Songjaroen, T.; Dungchai, W.; Henry, C. S.; Chailapakul, O.; Laiwattanapaisal, W. Anal. Chim. Acta 2013, 788, 39-45.

(28) Dungchai, W.; Chailapakul, O.; Henry, C. S. Anal. Chem. 2009, 81, 5821-5826.

(29) Maxwell, E. J.; Mazzeo, A. D.; Whitesides, G. M. MRS Bull. 2013, 38, 309-314.

(30) Mirica, K. A.; Weis, J. G.; Schnorr, J. M.; Esser, B.; Swager, T. M. Angew. Chem. Int. Ed. 2012, 51, 10740-10745.

(31) Santhiago, M.; Henry, C. S.; Kubota, L. T. Electrochim. Acta 2014, 130, 771-777.

(32) Adkins, J.; Boehle, K.; Henry, C. Electrophoresis 2015, 36, 1811-1824.

(33) Rungsawang, T.; Punrat, E.; Adkins, J.; Henry, C.; Chailapakul, O. Electroanalysis 2015, $27,1-8$.

(34) Apilux, A.; Dungchai, W.; Siangproh, W.; Praphairaksit, N.; Henry, C. S.; Chailapakul, O. Anal. Chem. 2010, 82, 1727-1732.

(35) Lan, W.; Maxwell, E. J.; Parolo, C.; Bwambok, D. K.; Subramaniam, A. B.; Whitesides, G. M. Lab Chip 2013, 13, 4103-4108.

(36) Lan, W.; Zou, X. U.; Hamedi, M. M.; Hu, J.; Parolo, C.; Maxwell, E. J.; Bu, P.; Whitesides, G. M. Anal. Chem. 2014, 86, 9548-9553. 
(37) Nemiroski, A.; Christodouleas, D. C.; Hennek, J. W.; Kumar, A. A; Maxwell, E. J.; Fernández-abedul, M. T.; Whitesides, G. M. Proc. Natl. Acad. Sci. U. S. A. 2014, 111, 1-6.

(38) Glavan, A. C.; Christodouleas, D. C.; Mosadegh, B.; Yu, H. D.; Smith, B. S.; Lessing, J.; Ferna, M. T.; Whitesides, G. M. Anal. Chem. 2014, 86, 11999-12007.

(39) Glavan, A. C.; Ainla, A.; Hamedi, M. M.; Fernández-Abedul, M. T.; Whitesides, G. M. Lab Chip 2016, 16, 112-119.

(40) Fosdick, S. E.; Anderson, M. J.; Renault, C.; Degregory, P. R.; Loussaert, J. A.; Crooks, R. M. Anal. Chem. 2014, 86, 3659-3666.

(41) Rattanarat, P.; Dungchai, W.; Cate, D.; Volckens, J.; Chailapakul, O.; Henry, C. S. Anal. Chem. 2014, 86, 3555-3562.

(42) Renault, C.; Anderson, M. J.; Crooks, R. M. J. Am. Chem. Soc. 2014, 136, 4616-4623.

(43) Scida, K.; Cunningham, J. C.; Renault, C.; Richards, I.; Crooks, R. M. Anal. Chem. 2014, $86,6501-6507$.

(44) Mirica, K. A.; Azzarelli, J. M.; Weis, J. G.; Schnorr, J. M.; Swager, T. M. Proc. Natl. Acad. Sci. U.S.A. 2013, 110, 3265-3270.

(45) Christodouleas, D. C.; Nemiroski, A.; Kumar, A. A.; Whitesides, G. M. Anal. Chem. 2015, 87, 9170-9178.

(46) Carrilho, E.; Martinez, A. W.; Whitesides, G. M. Anal. Chem. 2009, 81, 7091-7095.

(47) Yuen, P. K.; Goral, V. N. Lab Chip 2010, 10, 384-387.

(48) Yu, H. Y. E.; Agus, M.; Kellogg, M. D. Pediatr Diabetes 2011, 12, 649-655.

(49) Vallée-Bélisle, A.; Ricci, F.; Uzawa, T.; Xia, F.; Plaxco, K. W. J. Am. Chem. Soc. 2012, 134, 15197-15200.

(50) Yao, H.; Halsall, H. B.; Heineman, W. R.; Jenkins, S. H. Clin. Chem. 1995, 41, 591-598.

(51) Khorsand, F.; Darziani Azizi, M.; Naeemy, A.; Larijani, B.; Omidfar, K. Mol. Biol. Rep. 2013, 40, 2327-2334. 
(52) Kochius, S.; Magnusson, A. O.; Hollmann, F.; Schrader, J.; Holtmann, D. Appl. Microbiol. Biotechnol. 2012, 93, 2251-2264.

(53) Tang, Z.; Lee, J. H.; Louie, R. F.; Kost, G. J. Arch. Pathol. Lab. Med. 2000, 124, 11351140.

(54) Carver L. Bring Glucose Monitoring to New Levels through Integrated Sensor Design. IEEE Spectrum Multiphysics Simulation [Online], September 2015, p24-26. http:// www.comsol.com/offers/mphsim 15 (accessed December 21, 2015)

(55) Li, X.; Zwanenburg, P.; Liu, X. Lab Chip 2013, 13, 2609-2614.

(56) Siegel, A. C.; Phillips, S. T.; Dickey, M. D.; Lu, N.; Suo, Z.; Whitesides, G. M. Adv. Funct. Mater. 2010, 20, 28-35.

(57) Blees, M. K.; Barnard, A. W.; Rose, P. a.; Roberts, S. P.; McGill, K. L.; Huang, P. Y.; Ruyack, A. R.; Kevek, J. W.; Kobrin, B.; Muller, D. a.; McEuen, P. L. Nature 2015, 524, 204-207.

(58) Lamoureux, A.; Lee, K.; Shlian, M.; Forrest, S. R.; Shtein, M. Nat. Commun. 2015, DOI:10.1038/ncomms9092

(59) Nogi, M.; Komoda, N.; Otsuka, K.; Suganuma, K. Nanoscale 2013, 5, 4395-4399.

(60) Liu, H.; Crooks, R. M. J. Am. Chem. Soc. 2011, 133, 17564-17566.

(61) P. Yang, Z.-H. Lin, K. C. Pradel, L. Lin, X. Li, X. Wen, J.-H. He, Z. L. Wang, ACS Nano 2015, 9, 901-907.

(62) Scida, K.; Li, B.; Ellington, A. D.; Crooks, R. M. Anal. Chem. 2013, 85, 9713-9720.

(63) Luo, L.; Li, X.; Crooks, R. M. Anal. Chem 2014, 86, 12390-12397.

(64) Li, X.; Scida, K.; Crooks, R. M. Anal. Chem. 2015, 87, 9009-9015.

(65) Davidson, M. B. Ann. Intern. Med. 2003, 138, 517-523.

(66) Goldstein, D. E.; Little, R. R.; Lorenz, R. A.; Malone, J. I.; Nathan, D.; Peterson, C. M.; Sacks, D. B. Diabetes Care 2004, 27, 1761-1773. 
(67) Voulgari, C.; Tentolouris, N. Diabetes Technol. Ther. 2010, 12, 529-535.

(68) Bektas, F.; Eray, O.; Sari, R.; Akbas, H. Endocr. Res. 2004, 30, 395-402.

(69) Yeaw, J.; Lee, W. C.; Aagren, M.; Christensen, T. J. Manag. Care Pharm. 2012, 18, 2132.

(70) Kitabchi, A. E.; Umpierrez, G. E.; Miles, J. M.; Fisher, J. N. Diabetes Care 2009, 32, 13351343. 
Table of Content: Pop-up paper-based electrochemical device.

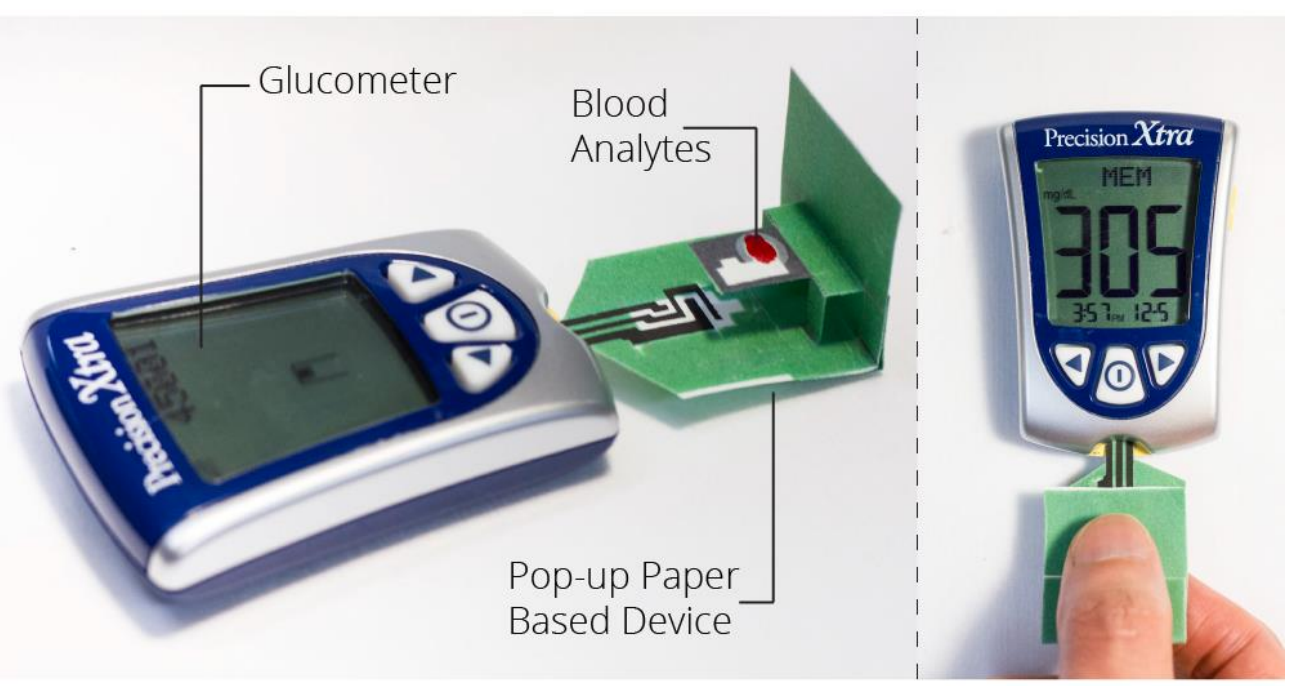

